\title{
Detailed noise statistics for an optically preamplified direct detection receiver
}

\author{
Danielsen, Søren Lykke; Mikkelsen, Benny; Durhuus, Terji; Jørgensen, Carsten; Stubkjær, Kristian
}

Published in:

Journal of Lightwave Technology

Link to article, DOI:

$10.1109 / 50.387817$

Publication date:

1995

Document Version

Publisher's PDF, also known as Version of record

Link back to DTU Orbit

Citation (APA):

Danielsen, S. L., Mikkelsen, B., Durhuus, T., Jørgensen, C., \& Stubkjær, K. (1995). Detailed noise statistics for an optically preamplified direct detection receiver. Journal of Lightwave Technology, 13(5), 977-981.

https://doi.org/10.1109/50.387817

\section{General rights}

Copyright and moral rights for the publications made accessible in the public portal are retained by the authors and/or other copyright owners and it is a condition of accessing publications that users recognise and abide by the legal requirements associated with these rights.

- Users may download and print one copy of any publication from the public portal for the purpose of private study or research.

- You may not further distribute the material or use it for any profit-making activity or commercial gain

- You may freely distribute the URL identifying the publication in the public portal 


\title{
Detailed Noise Statistics for an Optically Preamplified Direct Detection Receiver
}

\author{
Soeren Lykke Danielsen, Benny Mikkelsen, Terji Durhuus, Carsten Joergensen, and Kristian E. Stubkjaer
}

\begin{abstract}
We describe the exact statistics of an optically preamplified direct detection receiver by means of the moment generating function. The theory allows an arbitrary shaped electrical filter in the receiver circuit. The moment generating function (MGF) allows for a precise calculation of the error rate by using the inverse Fast Fourier transform (FFT). The exact results are compared with the usual Gaussian approximation (GA), the saddlepoint approximation (SAP) and the modified Chernoff bound (MCB). This comparison shows that the noise is not Gaussian distributed for all values of the optical amplifier gain. In the region from 20-30 dB gain, calculations show that the GA underestimates the receiver sensitivity while the SAP is very close to the results of our exact model. Using the MGF derived in the article we then find the optimal bandwidth of the electrical filter in the receiver circuit and calculate the sensitivity degradation due to inter symbol interference (ISI).
\end{abstract}

\section{INTRODUCTION}

$\mathbf{S}$ EVERAL approaches have been used to calculate sensitivities of optically preamplified direct detection receivers. Personick [1] used the Chernoff bound to evaluate an upper bound on the error rate assuming Gaussian distributed amplifier noise. Recently [2], the modified Chernoff bound (MCB) $[3],[4]$ has been used and Yamamoto uses the well known Gaussian approximation (GA) [5].

In this article a complete description of the detection process including the filtering in the electrical receiver circuit is given by means of the moment generating function (MGF). This allows for a precise calculation of the bit error rate using the inverse Fast Fourier Transform (FFT). We will refer to this method as MGF-FFT. For comparison the receiver sensitivities are estimated using the MCB, GA and the saddlepoint approximation (SAP) [6]. Assuming that the electrical filter in the receiver circuit consists of a perfect integrator, numerical calculations show that applying the SAP to the MGF one obtains a more correct approximation than is obtained with $\mathrm{GA}$ and MCB. That GA underestimates the bit error rate is also concluded in [7] where GA has been compared to the noncentral-negative-binomial (NNB) distribution. Finally, SAP is used to calculate sensitivities when the electrical filtering consists of a 3 rd order Butterworth filter.

\section{ReCEIVER MOMENT GenERATING FunCtION}

The receiver which is investigated here is the standard configuration shown schematically in Fig. 1. Pulses at rate

Manuscript received April 4, 1994; revised December 28, 1994.

The authors are with the Center for Broadband Telecommunication, Electromagnetics Institute, Technical University of Denmark, DK-2800 Lyngby, Denmark.

IEEE Log Number 9409607.

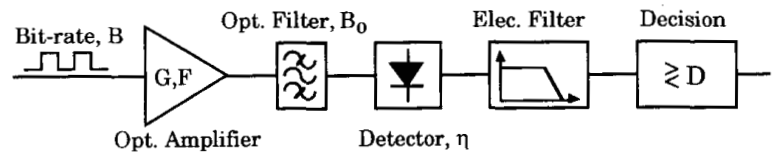

Fig. 1. Direct detection receiver configuration.

$B=1 / T, T$ being the bit period, arrive at the optical preamplifier that is described by the noise figure, $F$, and the gain, $G$. The coupling efficiency at the input of the amplifier is $\Gamma_{i n}$. The amplifier is followed by a square shaped optical filter of bandwidth $B_{o}$. Note that if no filter is used, $B_{o}$ is the bandwidth of the amplifier. A pin diode with the quantum efficiency $\eta$ is followed by an electrical filter with the impulse response $h_{R}(t)$ that shapes the signal. This signal is then sampled and a decision is made, whether a mark or a space has been received. The loss between the output of the amplifier and the pin diode, i.e., the coupling loss at the amplifier output and the optical filter loss, is $\Gamma_{\text {out }}$.

Following [8] the distribution of the photon number at the preamplifier output is taken to be that of the population of a birth-death-immigration (BDI) [9]. Furthermore, we assume a Poisson distributed input signal to the optical amplifier for which the probability that exactly $N$ photons, couples to the amplifier in an infinitesimal time interval $\Delta t$ centered around the time $t$ is given as $\left(\Lambda \Gamma_{i n}\right)^{N} \exp \left(-\Lambda \Gamma_{i n}\right) / N$ !. Here $\Lambda=\lambda(t) \Delta t$ and $\lambda(t)$ is the average rate at which the photons arrive. This rate equals $p(t) / h v$, where $p(t)$ is the incident signal power while $h$ and $v$ are Planck's constant and the light frequency, respectively. As before, $\Gamma_{i n}$ is the coupling efficiency at the amplifier input. From the BDI process we can derive a partial differential equation, as shown in the appendix, governing the MGF of the number of photons that leave the optical amplifier

$$
\begin{aligned}
& \frac{\partial M_{N}(s, t)}{\partial t}=\sigma_{s p}\left(e^{s}-1\right) M_{N}(s, t) \\
& +\left(\sigma_{e}-\sigma_{a} e^{-s}\right)\left(e^{s}-1\right) \frac{\partial M_{N}(s, t)}{\partial s}
\end{aligned}
$$

where $\sigma_{e}$ is the stimulated emission rate per photon. $\sigma_{a}$ is the absorption rate per photon and $\sigma_{s p}=\sigma_{e} B_{o} \Delta t$ is the spontaneous emission rate per unit time. $M_{N}(s, t)$ is the moment generating function that corresponds to the distribution of photons at the output of the amplifier when the number of photons that couples to the amplifier input during the time, $\Delta t$, centered around $t$, is $N$. 
The solution to the differential equation above is given as

$$
\begin{array}{r}
M_{N}(s, t)=\left[\frac{1}{1+N_{o}\left(1-e^{s}\right)}\right]^{B_{o} \Delta t} \\
\cdot\left[\frac{1+\left(N_{o}-G\right)\left(1-e^{s}\right)}{1+N_{o}\left(1-e^{s}\right)}\right]^{N}
\end{array}
$$

where $N_{o}=F / 2(G-1)$. The noise figure, $F$, is given as twice the spontaneous inversion parameter, $n_{s p}=\sigma_{e} /\left(\sigma_{e}-\sigma_{a}\right)$, and the gain, $G$, is given as $\exp \left(\left(\sigma_{e}-\sigma_{a}\right) \tau_{t}\right)$ when $\sigma_{e}$ and $\sigma_{a}$ are assumed time independent. $\tau_{t}$ is the transit time of the amplifier and it should be noted that the time, $t$, in (2) corresponds to the time at which the photons leaves the amplifier i.e., the conversion $t \rightarrow t+\tau_{t}$ is made going from (1) to (2). Assuming that $N$ is Poisson distributed, as described above, we can find the MGF of the random variable, $Y$, which represents the number of photons at the amplifier output

$$
\begin{aligned}
M_{Y}(s, t)= & \sum_{N=0}^{+\infty} M_{N}(s, t)\left(\Lambda \Gamma_{i n}\right)^{N} \frac{\exp \left(-\Lambda \Gamma_{i n}\right)}{N !} \\
= & {\left[\frac{1}{1+N_{o}\left(1-e^{s}\right)}\right]^{B_{\circ} \Delta t} } \\
& \cdot \exp \left[-\Lambda \Gamma_{i n} \frac{G\left(1-e^{s}\right)}{1+N_{o}\left(1-e^{s}\right)}\right] .
\end{aligned}
$$

In the above equation, $G \Lambda \Gamma_{i n}$ signifies the average number of signal photons while $N_{0}$ is the average number of spontaneously emitted photons per mode. To include the loss between the amplifier output and the detector, we simply multiply these average numbers by the loss factor, $\Gamma_{\text {out }}$. Similarly, the quantum efficiency of the detector is accounted for by multiplying by $\eta$. To include the electrical filter response, $h_{R}$, of the detector in the expression for the MGF, we do as follows: the time axis is divided into infinitesimal intervals, $\Delta t$, centered around $t_{j}$. If a constant value of $j, k$ photons leave the pre-amplifier at time $t_{j}$, then ideally $k$ electron counts are received by the electrical circuit. When deducing the moment generating function, we treat these received electron counts as independent pulses passing the receiver circuit at time $t_{j}$. Therefore, they are weighted as $\exp \left(s h_{R}\left(t_{0}-t j\right) k\right)$ instead of $\exp (\mathrm{sk})[10]$ where $t_{0}$ is the sampling time. Thereby, the MGF at the sampling time $t_{0}$ corresponding to the average number of electron counts arriving in the interval $\Delta t$ centered around $t_{j}$ is written as

$$
\begin{aligned}
M_{Y}\left(s, t_{0}, t_{j}\right) & =\sum_{k=0}^{\infty} P_{y}(k) e^{k s h_{R}\left(t_{0}-t_{j}\right)} \\
& =M_{Y}\left(s h_{R}\left(t_{0}-t_{j}\right), t_{j}\right)
\end{aligned}
$$

where $P_{y}(k)$ is the photon number distribution at the amplifier output.

Since the electron amounts are statistically independent the total MGF at sample time $t_{0}$ is the product of all the MGF each covering different time intervals $\Delta t$

$$
M_{Y}\left(s, t_{0}\right)=\prod_{j} M_{Y}\left(s, t_{0}, t_{j}\right)
$$

which leads to

$$
\begin{aligned}
& M_{Y}\left(s, t_{0}\right) \\
& =\exp \left\{-2 B_{o} \int_{-\infty}^{+\infty} \ln \left[1+\Gamma_{\text {out }} \eta N_{o}\left(1-e^{s h_{R}\left(t_{0}-\tau\right)}\right)\right] d \tau\right\} \\
& \quad \cdot \exp \left\{-\frac{G \Gamma_{i n} \Gamma_{\text {out }} \eta}{h \nu} \int_{-\infty}^{+\infty} \sum_{i} b_{i} h_{p}(\tau-i T)\right. \\
& \left.\cdot \frac{1-e^{s h_{R}\left(t_{0}-\tau\right)}}{1+\Gamma_{\text {out }} \eta N_{o}\left(1-e^{s h_{R}\left(t_{0}-\tau\right)}\right)} d \tau\right\}
\end{aligned}
$$

In (6) the signal power incident upon the preamplifier is given as $\sum_{i} b_{i} h_{p}(t-i T)$ where $h_{p}(t)$ is the optical pulse shape normalized so that $\int_{-\infty}^{+\infty} h_{p}(t) d t=1 . b_{i}$ is the energy in the $i$ th bit, that is, either the energy in a binary mark, $E_{1}$, or in a binary space, $E_{0}$. The relation $r=E_{0} / E_{1}$ defines the extinction ratio. The factor 2 in the first part of the expression accounts for the fact that amplifier noise is added in two polarization's.

It should be noted that by series expansion of the expression (6) the same noise variance components as those of Yamamoto [5] can be derived.

\section{Conditioned MGF of RANDOM PulSE-SEQuenCE}

With (6) we have a MGF for an optical preamplifier direct detection receiver that includes a filter in the receiver. To complete the model we modify (6) to allow for random bit sequences. Assuming a sequence consisting of $2 n+1$ binary symbols with equal probability for marks and spaces, the probability of a given sequence is $1 / 2^{(2 n+1)}$. Averaging over all possible sequence relation's and with the $b_{i}$ being independent and identically distributed random variables we get by conditioning $b_{0}$

$$
\begin{aligned}
& M_{Y}\left(s, t_{0} \mid b_{0}=E_{q}\right) \\
& =\exp \left\{-2 B_{o} \int_{-\infty}^{+\infty} \ln \left[1+\Gamma_{\text {out }} \eta N_{o}\left(1-e^{s h_{R}\left(t_{0}-t\right)}\right)\right] d t\right\} \\
& \cdot \exp \left\{E_{q} H_{0}\left(s, t_{0}\right)\right\} \\
& \cdot \frac{1}{2^{2 n}} \prod_{i=-2 n}^{i=-1}\left\{\exp \left[E_{1} H_{i}\left(s, t_{0}\right)\right] \exp \left[r E_{1} H_{i}\left(s, t_{0}\right)\right]\right\} \\
& q=0,1
\end{aligned}
$$

where

$$
\begin{aligned}
H_{i}\left(s, t_{0}\right)=-\frac{G \Gamma_{\text {in }} \Gamma_{\text {out }} \eta}{h \nu} \int_{-\infty}^{+\infty} h_{p}(\tau-i T) \\
\cdot \frac{1-e^{s h_{R}\left(t_{0}-\tau\right)}}{1+\Gamma_{\text {out }} \eta N_{o}\left(1-e^{s h_{R}\left(t_{0}-\tau\right)}\right)} d \tau .
\end{aligned}
$$

Equations (7) and (8) give a complete statistical description for the number of electron counts at the decision circuit due to the optical signal. In addition to these electron counts the electrical circuit itself produces thermal noise which we assume to be Gaussian distributed. The additive Gaussian noise 
at the input to the decision circuit is signal independent and is therefore modelled as a random variable $X$ with MGF [4]

$$
\begin{gathered}
M_{X}(s)=\exp \left(\frac{s^{2} \sigma^{2}}{2}\right), \\
\sigma^{2}=\frac{N_{t h}}{B e^{2}} \int_{-\infty}^{\infty}\left|H_{R}(f)\right|^{2} d f .
\end{gathered}
$$

Above $\sigma^{2}$ is the Gaussian noise variance so that $\sigma$ equals the number of thermal electron counts generated in $T$ sec. $f$ is the frequency normalized to the signal rate $B$ and $H_{R}(f)$ is the Fourier transform of $h_{R}(t)$ while $e$ is the electron charge. $N_{t h}$ is the noise spectral density of the receiver circuit, chosen to give a back to back sensitivity of $-21 \mathrm{dBm}$ at $B=10$ Gbit/s $(r=0)$.

At this point, $Y$, is the random variable that represents the number of electron counts due to the filtered signal and noise originating from the optical preamplifier. $X$ is the random variable that represents the thermal noise generated in the electrical receiver circuit. Letting the random variable, $Z$, represent the number of electron counts at the input to the decision circuit, $Z$ results by adding $Y$ and $X$. Therefore the MGF of $Z$ for marks and spaces can be written as $M_{Z_{0,1}}(s)=M_{Y}\left(s, t_{0} \mid b_{0}=E_{0,1}\right) M_{X}(s)$ where the time dependence of $M_{Z}$ has been suppressed for simplification.

\section{SADDLEPOINT APPROXIMATION AND UPPER BOUND (MCB) ON THE Average ERror Probability}

The errors at the decision occur with the probability $B E R=p_{0} P_{e 0}+p_{1} P_{e 1}$ where $p_{0,1}$ are a priori probabilities for marks and spaces taken to be a half. $P_{e 0}$ and $P_{e 1}$ are conditional error probabilities that can be found directly from the MGF using the FFT or by approximations such as MCB and SAP.

$P_{e 0}$ and $P_{e 1}$ can be written as

$$
\begin{aligned}
& P_{e 0}=\int_{D}^{+\infty} P_{Z_{0}}(z) d z \\
& P_{e 1}=\int_{-\infty}^{D} P_{Z_{1}}(z) d z
\end{aligned}
$$

where $D$ is the decision threshold and $P_{Z 0}(z)$ and $P_{Z 1}(z)$ are the conditional probability density functions that can be found from (7)-(9) by using the inverse Laplace transform. Carrying out the integration in (10.1-2) gives

$$
\begin{array}{r}
P_{e q}=(-1)^{q} \frac{1}{2 \pi i} \int_{c_{q}-i \infty}^{c_{q}+i \infty} \frac{1}{s} M_{z_{q}}(s) e^{-s D} d s \\
q=0,1 ; c_{0}>0, c_{1}<0
\end{array}
$$

$c_{0}$ and $c_{1}$ in (11) are chosen to be the saddlepoints of the integrals so that the contour of the integrals passes through these saddlepoints. By series expansion of the integrals we get the saddlepoint approximation [6]

$$
P_{e q} \cong\left[2 \pi \Psi_{q}^{\prime \prime}\left(s_{q}\right)\right]^{-1 / 2} \exp \left[\Psi_{q}\left(s_{q}\right)\right] \quad q=0,1
$$

where the functions $\Psi_{0,1}$ are defined as

$$
\exp \left(\Psi_{q}(s)\right)=(-1)^{q} \frac{1}{s} M_{Z_{q}}(s) \exp (-s D) \quad q=0,1
$$

in which $s_{0,1}$ in (12) are the saddlepoints that are found by setting the derivatives of $\Psi_{0}$ and $\Psi_{1}$ to zero. Finding these $s$-values and the optimal threshold, enables an approximation of the error rate.

The MCB is found by evaluating the upper bound of the integrals in (11). This is done by using that the absolute value of an integral never exceeds the value of the absolute integral. The result is [3], [4]

$$
P_{e q} \leq M C B_{q}=\frac{1}{\sqrt{2 \pi} \sigma} \exp \left[\Psi_{q}\left(s_{q}\right)\right] \quad q=0,1 .
$$

Again this bound is minimized with respect to $s_{0,1}$ and $D$.

\section{CAlculations and Discussion}

In case the electrical filter is that of an ideal integrator of duration $T$, sensitivities have been calculated using all the methods outlined above, i.e., SAP, MCB, GA and MGF-FFT. The latter will also be referred to as the exact method. The FFT used in the calculations can determine the probability density function at photon probabilities down to $10^{-20}$ which is considered acceptable to predict $B E R$ of $10^{-9}$. A noise figure of $3 \mathrm{~dB}$ is assumed for the optical amplifier and a signal rate of $10 \mathrm{Gbit} / \mathrm{s}(r=0)$ is used in all cases. System losses are neglected so that $\Gamma_{\text {in }}=1$ and $\Gamma_{\text {out }}=1$ while $\eta=1$. The optical pulse shape is taken to be a NRZ sequence. Two values of the optical filter bandwidth have been investigated. For optical filter bandwidths of 10 and $125 \mathrm{GHz}$, the mean number of photons necessary to achieve a $B E R$ of $10^{-9}$ are given in Fig. 2(a) and 2(b), respectively, as a function of the optical amplifier gain.

The exact method and the SAP are almost identical for all gain values. For large gain values the exact sensitivities and those found by using SAP both converge towards 41 and 62 counts for optical filter bandwidths of 10 and $25 \mathrm{GHz}$, respectively.

The MCB is more complex because the factor $\exp \left(s^{2} \sigma^{2} / 2\right) /(s \sigma)$, which is found by inserting (9) and (13) into (14), is gain dependent since the absolute values of $s_{0,1}$ decreases when $G$ is increased. Hence, for larger gain values $s \sigma$ is less than one and, consequently, the bit error rate calculated from the MCB will increase.

The results indicate three characteristic gain regions for the Gaussian approximation: low gain values $(0-20 \mathrm{~dB})$, medium gain values $(20-30 \mathrm{~dB})$ and gain values larger than $30 \mathrm{~dB}$. For low amplifier gain values the Gaussian distributed thermal noise dominates the optical amplifier noise. As a result the curves in Fig. 2(a) and 2(b) are identical in this region. For amplifier gain values between 20 and $30 \mathrm{~dB}$ (typical gain values for an optical amplifier) the estimate of GA is different from the prediction of the exact method. In this gain region it is seen that GA predicts a higher receiver sensitivity although the worst case deviation is only 9 counts $(0.6 \mathrm{~dB})$ when $B_{o}=10 \mathrm{GHz}$ and 4 counts $(0.23 \mathrm{~dB})$ when $B_{o}=$ $125 \mathrm{GHz}$. Comparison of Fig. 2(a) and 2(b) shows that the difference between the GA and the exact method reduces for the larger optical filter bandwidth. For large gain values the GA converges towards 42 and 64 counts for optical filter bandwidths of 10 and $125 \mathrm{GHz}$, respectively. As in [7] we 


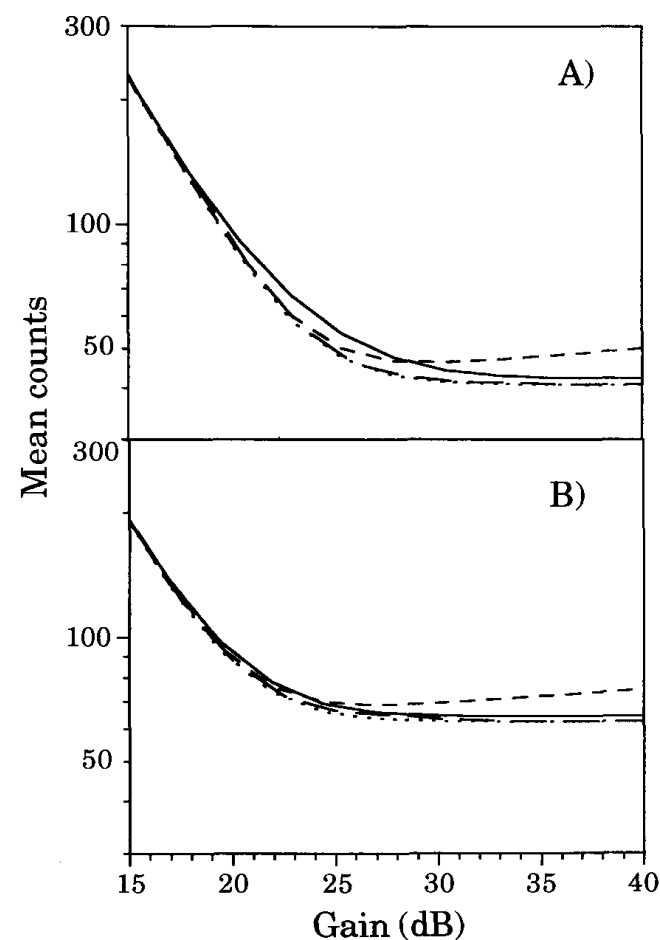

Fig. 2. Sensitivities expressed by the mean number of counts at $10 \mathrm{Gbit} / \mathrm{s}$ and an optical amplifier noise figure of $3 \mathrm{~dB}$. (a) is for $B_{0}=10 \mathrm{GHz}$ while (b) is for $B_{o}=125 \mathrm{GHz}, \eta=1, r=0$ and the filter is that of an ideal integrator. Results are shown for SAP $(\cdots)$, Gauss $(-)$, MCB $(-\cdots)$, and exact method (-- $)$.

therefore conclude that even in the limit (many counts) the distribution of electron counts is not that of a Gaussian random variable, despite of the predictions of the central limit theorem.

The discussion above and Fig. 2(a) and 2(b) shows that SAP is a good approximation while for gain values larger than $20 \mathrm{~dB}$, MCB clearly underestimates the sensitivity. Since the complexity of using the SAP and the MCB is the same, SAP is the preferable method.

The advantage of the GA is its simplicity. However, in more complex systems such as wavelength division multiplexing (WDM) systems where crosstalk is present the GA becomes more complex while the complexity of the SAP and the MCB remains the same: for statistically independent signals the MGF for each of these signals should simply be multiplied to form the MGF that is used in the SAP and the MCB.

Furthermore, the GA cannot predict the errors caused by nonideal electrical filtering such as intersymbol interference (ISI). The (7)-(9) presented in this article do on the other hand allow for any electrical filtering.

As an example of using (7)-(9) with a more realistic electrical filter we have calculated sensitivities by implementing a 3rd order Butterworth filter in the MGF. The electrical filter bandwidth is optimized by calculating the sensitivities of the receiver when the filter bandwidth is swept from $0.6-0.9$ times the bit rate, $B$, with a step of $0.005 B$. This is done for the case of an optical preamplifier gain of $40 \mathrm{~dB}$ and a signal power of $-41 \mathrm{dBm}$ coupled to the preamplifier. The sampling time is

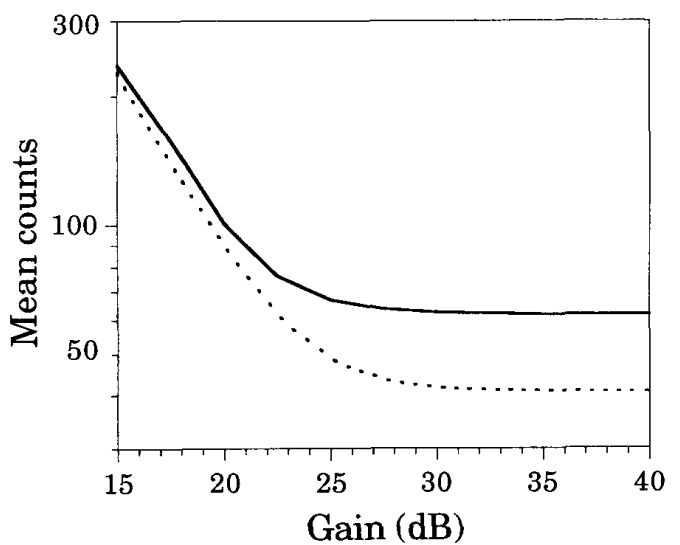

Fig. 3. Comparison of sensitivities using 3rd order Butterworth filter ( - ) and an ideal integrator $(\cdots)$, respectively. $B=10 \mathrm{Gbit} / \mathrm{s}, B_{o}=125 \mathrm{GHz}$, $\eta=1, F=3 \mathrm{~dB}$ and $r=0$.

chosen as the maximum point of the convolution of $h_{p}(t)$ and $h_{R}(t)$ and at the sampling time the convolution is normalized to one. This leads to an optimal filter bandwidth of 0.695 times the bit rate. In Fig. 3 sensitivities using the Butterworth filter and an ideal integrator are compared for an optical bandwidth of $10 \mathrm{GHz}$ and a signal rate of $10 \mathrm{Gbit} / \mathrm{s}(r=0)$. The length of the bit sequence is $2^{7}-1$. A degradation of the detection process due to the non ideal electrical filtering is observed and the difference between the curves is as much as $1 \mathrm{~dB}$ at low gain values and $1.8 \mathrm{~dB}$ at high gain values.

\section{CONCLUSION}

A MGF describing the exact statistics of an optically preamplified direct detection receiver including an arbitrary electrical filter have been derived. By applying the inverse FFT, SAP and $\mathrm{MCB}$ to the receiver MGF, exact and approximated receiver sensitivities for different values of the optical amplifier gain have been compared. With a perfect extinction ratio, a bit rate of $10 \mathrm{Gbit} / \mathrm{s}$, a noise figure of $3 \mathrm{~dB}$ and using an ideal integrator as the electrical filter we have shown that the SAP gives almost exact results; the deviation from the exact results are at most 1 and 2 photon(s)/bit. With an optical filter bandwidth of 10 and $125 \mathrm{GHz}$ the SAP underestimates the receiver sensitivity by 0.09 and $0.07 \mathrm{~dB}$, respectively.

Inside the gain region from $20-30 \mathrm{~dB}$ the GA predicts sensitivities that are up to 0.6 and $0.23 \mathrm{~dB}$ worse than the sensitivities found by using the MGF-FFT for optical filter bandwidths of 10 and $125 \mathrm{GHz}$, respectively, while outside this gain region the GA is very close to the exact results.

The moment generating function derived in this article makes it possible to find the optimal electrical filter bandwidth giving the best sensitivity. With a bit rate of $10 \mathrm{Gbit} / \mathrm{s}$ the optimal bandwidth of a 3rd order Butterworth filter is found to be 0.695 times the bit rate assuming a perfect extinction ratio, a noise figure of $3 \mathrm{~dB}$ and no loss in the system. Using this filter bandwidth, the effect of ISI is analyzed by comparing sensitivities using the Butterworth filter and an ideal integrator. For an optical filter bandwidth of $10 \mathrm{GHz}$ the sensitivity degradation due to ISI is found to be $1 \mathrm{~dB}$ at low gain values and $1.8 \mathrm{~dB}$ at gain values higher than $30 \mathrm{~dB}$. 
Finally we emphasize that the methods based on the moment generating functions described here is well suited for describing crosstalk in WDM networks since the MGF of a sum of statistically independent signals is simply the product of the each individual MGF.

\section{APPENDIX \\ DERIVATION OF (1)}

In the following the partial differential (1) describing the moment generating function of the number of photons that leave the optical amplifier is derived.

As in [8] the output of the optical amplifier is modelled as a BDI process with the following birth, death, and emigration rates:

1) $\sigma_{e}$ is the stimulated emission rate per photon. When modelling the BDI process this is therefore the birth rate.

2) $\sigma_{a}$ is the absorption rate per photon. When modelling the BDI process this is therefore the death rate.

3) $\sigma_{s p}$ is the spontaneous emission rate per unit time. Since the spontaneous emission rate per mode equals the stimulated emission rate per photon, and since the number of temporal modes in the time interval $\Delta t$ is $B_{o} \Delta t, \sigma_{s p}$ equals $\sigma_{e} B_{o} \Delta t$. When modelling the BDI process $\sigma_{s p}$ is the emigration rate.

Given that $N$ photons exist at time $t, P_{N, m}(t+\delta t)$, denote the probability that $m$ photons exist at time $t+\delta t$. Using $\sigma_{e}$, $\sigma_{a}$ and $\sigma_{s p}, P_{N, m}(t+\delta t)$ is found from

$$
\begin{aligned}
P_{N, m}(t+ & \delta t) \\
= & \left\{1-\left[\left(\sigma_{e} m+\sigma_{a} m+\sigma_{s p}\right) \delta t+o(\delta t)\right]\right\} P_{N, m}(t) \\
& +\left\{\left[\sigma_{e}(m-1)+\sigma_{s p}\right] \delta t+o(\delta t)\right\} P_{N, m-1}(t) \\
& +\left\{\sigma_{a}(m+1) \delta t+o(\delta t)\right\} P_{N, m+1}(t) \\
& +o(\delta t)
\end{aligned}
$$

where $o(\delta t) / \delta t \rightarrow 0$ as $\delta t \rightarrow 0$.

Letting $\delta t \rightarrow 0$ we get

$$
\begin{aligned}
\frac{d P_{N, m}(t)}{d t}= & -\left[\left(\sigma_{e}+\sigma_{a}\right) m+\sigma_{s p}\right] P_{N, m}(t) \\
& +\left[\sigma_{e}(m-1)+\sigma_{s p}\right] P_{N, m-1}(t) \\
& +\left[\sigma_{a}(m+1)\right] P_{N, m+1}(t) .
\end{aligned}
$$

Multiplying with $e^{s m}$ and summing over all $m$ greater than or equal to zero gives

$$
\begin{aligned}
\sum_{m} \frac{d P_{N, m}(t)}{d t} e^{s m} \\
=-\sum_{m}\left(\sigma_{e}+\sigma_{a}\right) m P_{N, m}(t) e^{s m}-\sigma_{s p} \sum_{m} P_{N, m}(t) e^{s m} \\
\quad+e^{s} \sum_{m} \sigma_{e}(m-1) P_{N, m-1}(t) e^{s(m-1)} \\
\quad+e^{s} \sum_{m} \sigma_{s p} P_{N, m-1}(t) e^{s(m-1)} \\
\quad+e^{-s} \sum_{m} \sigma_{a}(m+1) P_{N, m+1}(t) e^{s(m+1)}
\end{aligned}
$$

Introducing $m^{\prime}=m-1, m^{\prime \prime}=m+1$ and recognizing the following:

$$
\begin{aligned}
M_{N}(s, t) & =\sum_{m} P_{N, m}(t) e^{s m} \\
\frac{\partial M_{N}(s, t)}{\partial s} & =\sum_{m} m P_{N, m}(t) e^{s m} \\
\frac{\partial M_{N}(s, t)}{\partial t} & =\sum_{m} \frac{d P_{N, m}}{d t} e^{s m} .
\end{aligned}
$$

Equation (A3) can be rewritten into (1)

$$
\begin{aligned}
\frac{\partial M_{N}(s, t)}{\partial t}= & \sigma_{s p}\left(e^{s}-1\right) M_{N}(s, t) \\
& +\left(\sigma_{e}-\sigma_{a} e^{-s}\right)\left(e^{s}-1\right) \frac{\partial M_{N}(s, t)}{\partial s}
\end{aligned}
$$

\section{REFERENCES}

[1] S. D. Personick, "Applications for quantum amplifiers in simple digital optical communication systems," Bell Syst. Tech. J., vol. 52, no. 1, pp. $117-133,1973$

[2] R. S. Fayth and J. J. O'Reilly, "Comprehensive moment generating function characterisation of optically preamplified receivers," IEE Proc.,pt. J, vol. 137, no. 6, pp. 391-396, 1990.

[3] V. K. Prabhu, "Modified Chernoff bounds for PAM systems with noise and interference," IEEE Trans. Info. Theory, vol. 28, no. 1, pp. 95-99, 1982.

[4] J. J. O'Reilly and J. R. F. Da Rocha, "Improved error probability evaluation methods for direct detection optical communication systems," IEEE Trans. Info. Theory, vol. 33, no. 6, pp. 838-848, 1987.

[5] Y. Yamamoto, "Noise and error rate performance of semiconductor laser amplifiers in PCM-IM optical transmission systems," IEEE J. Quantum Electron., vol. 16, no. 10, pp. 1073-1081, 1980.

[6] C. W. Helstrom, "Approximate evaluation of detection probabilities in radar and optical communications," IEEE Trans. Aerospace and Electron. Systems, vol. 14, no. 4, pp. 630-640, 1978.

[7] T. Li and M. C. Teich, "Bit-error rate for a lightwave communication system incorporating an Erbium doped fibre amplifier," Electron. Lett., vol. 27, no. 7 , pp. 598-600, 1991.

[8] K. Shimoda, H. Takahashi, and C. H. Townes, "Fluctuations in amplification of quanta with application to Maser amplifiers," J. Phys. Soc. Jpn., vol. 12, no. 6, pp. 686-700, 1957.

[9] S. M. Ross, Introduction to Probability Models, 3rd ed. San Diego: Academic, 1985.

[10] K. W. Cattermole and J. J. O'Reilly, Mathematical Topics in Telecommunications, Optimisation Methods in Electronics and Communications, vol. 1. Pentech, 1984.

Soeren Lykke Danielsen, photograph and biography not available at the time of publication.

Benny Mikkelsen, photograph and biography not available at the time of publication.

Terji Durhuus, photograph and biography not available at the time of publication.

Carsten Joergensen, photograph and biography not available at the time of publication.

Kristian E. Stubkjaer, photograph and biography not available at the time of publication. 